\title{
Monte Carlo simulation of skin multispectral autofluorescence
}

\author{
Anastasia O. Ustinova, Ivan A. Bratchenko, and Dmitry N. Artemyev* \\ Samara National Research University, 34 Moskovskoye shosse, Samara 443086, Russia \\ *e-mail: artemev.dn@ssau.ru
}

\begin{abstract}
This work is devoted to the simulation of human skin autofluorescence in different spectral ranges. Analytical review was performed for selecting the main endogenous fluorophores with the greatest contribution to the skin fluorescence: tryptophan, tyrosine, collagen, melanin, elastin, lipofuscin, protoporphyrin IX, NADH, FAD. It was necessary to set parameters for autofluorescence modeling, such as the absorption/emission spectra of fluorophores, molar concentration, molar extinction coefficient, and quantum yield. The six-layer skin model was designed in the TracePro software and autofluorescence was simulated when excited at different wavelengths in the middle UV (270-300 nm), near UV $(330-360 \mathrm{~nm})$ and visible $(400-450 \mathrm{~nm})$ spectral ranges. The simulation results were compared with the experimental results of other authors. The principal distinctive factor of this work is the simulation of the human skin autofluorescence excited in different spectral ranges. (C) 2019 Journal of Biomedical Photonics \& Engineering.
\end{abstract}

Keywords: autofluorescence; Monte Carlo modeling; skin model; skin fluorophores; optical diagnostics.

Paper \#3326 received 2 May 2019; revised manuscript received 8 Jun 2019; accepted for publication 15 Jun 2019; published online 30 Jun 2019. doi: 10.18287/JBPE19.05.020306.

\section{Introduction}

Autofluorescence (AF) of the skin is the fluorescent radiation of natural fluorophores inside the tissue excited by absorption radiation with a specific wavelength. Mathematical modeling of AF makes it possible to predict the behavior of real systems and solve design problems without carrying out real experiments [1].

It is possible to reproduce the spectral-optical properties of a biological object in a model experiment. In this case, the model can simulate the spatial structure, composition, and localization of the fluorophores in a real tissue. It is possible to change the parameters of the skin model: the component concentration, the initial radiation conditions, tracking changes in the fluorescence spectra of an object. The method of AF spectroscopy (AFS) is based on the fluorescence spectra registration of endogenous biomarkers. It is known that tumors of the skin, the oral cavity membranes, the gastrointestinal tract, have a number of specific $\mathrm{AF}$ spectra, which can be an additional diagnostic parameter for the doctor [2]. However, the AFS method has a number of unresolved problems. Many fluorophores have similar or overlapping absorption and emission spectra, with the result that the tissue fluorescence has a complex spectral composition. In this regard, there is uncertainty with the excitation wavelength and with the emission wavelength for each specific fluorophore [3]. This paper demonstrates the dependences of the shape and intensity of AF spectra by excitation radiation at different wavelengths in three spectral ranges. The aim of this work is AF simulation of human skin model for interpreting experimental spectra (estimating the fluorophores contributions) and assessment of various tissue states (inflammation, neoplasm, etc.).

\section{Modeling of light propagation in tissues by Monte Carlo method}

The model of radiation propagation in biological tissue was used to describe and predict the effect of fluorophores on the AF signal. Fluorescence simulation was carried out by the Monte Carlo method using the 
Table 1 Fluorescent properties of skin components.

\begin{tabular}{lccc}
\hline \multicolumn{1}{c}{ Fluorophore } & Quantum yield, $\eta_{F}$ & $\begin{array}{c}\text { Peak of molar extinction, } \varepsilon_{\lambda} \\
{[1 / \mathrm{mol} \cdot \mathrm{cm}]}\end{array}$ & $\begin{array}{c}\text { Molar concentration, } C_{a b,} \\
{[\mathrm{~mol} / \mathrm{l}]}\end{array}$ \\
\hline Elastin [8, 11] & 0.25 & 28400 & $6 \cdot 10^{-5}$ \\
Lipofuscin [6, 13] & 0.02 & 11500 & $10^{-3}$ \\
Protoporphyrin IX [6, 14] & 0.06 & 171000 & $1.8 \cdot 10^{-6}$ \\
NADH [8, 12] & 0.019 & 3400 & $2 \cdot 10^{-3}$ \\
FAD [8] & 0.03 & 11300 & $5.3 \cdot 10^{-5}$ \\
Melanin [7] & 0.003 & 2100 & $0.88 \cdot 10^{-2}$ \\
Tyrosine [9] & 0.13 & 1398 & $2.5 \cdot 10^{-4}$ \\
Tryptophan [8, 15] & 0.12 & 5509 & $5 \cdot 10^{-3}$ \\
Collagen [10] & 0.3 & 52940 & $1.8 \cdot 10^{-3}$ \\
\hline
\end{tabular}

TracePro Expert software environment - 6.0.2, which is designed to analyze radiation propagation in optical systems. This method takes into account absorption and scattering throughout the optical path of a photon through a turbid medium.

The Monte Carlo method is a computational method that includes a random sample of a physical quantity. It has become a popular tool for simulating radiation transfer in tissues, since it provides an accurate solution to the problem of radiation transfer in turbid media with a complex structure. The method is able to solve the radiation transfer equation with any required accuracy, if the necessary computing resources are available. For this reason, this method is considered as a gold standard for modeling the light transfer in tissues. The results of Monte Carlo simulations are often used as a reference for testing other less rigorous methods [4].

The optical scheme in the proposed model consists of a laser source, a detector and a six-layer skin model. The source and detector are perpendicular to each other to avoid illumination of the detector channel. The size of the detector was comparable to the size of the object for the efficient registration of the fluorescent signal. The number of incident photons to excite fluorescence in the simulation was 10,000 , which led to the generation of more than $10^{6}$ fluorescent photons. For such amount, the correct result was achieved, since with a larger amount the AF spectrum remained almost unchanged. The tracing process at a single excitation wavelength was about 5-6 minutes.

\section{Skin model for multispectral AF measurement}

The optical properties such as absorption and scattering coefficients, anisotropy coefficient and refractive indices for each skin layer depending on used wavelength were chosen from the book edited by Prof. Tuchin [5]. The model skin area was selected with the size of $40 \mathrm{~mm} \times 40 \mathrm{~mm}$. The thickness of individual skin layers: the stratum corneum - $10 \mu \mathrm{m}$, the epidermis $-100 \mu \mathrm{m}$, the papillary dermis $-200 \mu \mathrm{m}$, the surface plexus of microvessels $-100 \mu \mathrm{m}$, the reticular dermis $-600 \mu \mathrm{m}$ and the deep plexus of the vessels $200 \mu \mathrm{m}$.

Fluorophores were characterized by quantum yield, molar extinction coefficient, and molar concentration, since the absorption and fluorescence intensity mainly depends on these parameters as [5]:

$$
\begin{aligned}
& I(\lambda)=I_{0} \exp \left(-\mu_{a} d\right)=I_{0} 10^{-\varepsilon_{\lambda} c_{a b} d}, \\
& I_{F}(\lambda)=I_{0}\left[1-10^{-\varepsilon_{\lambda} c_{a b} d}\right] \eta_{F} \Omega / 4 \pi
\end{aligned}
$$

where $I(\lambda)$ is the intensity of the transmitted light, $I_{0}$ is the intensity of the incident light, $\mu_{a}$ is the absorption coefficient, $d$ is the layer thickness, $\varepsilon_{\lambda}$ is the molar extinction coefficient, $C_{a b}$ is the molar concentration, $\eta_{F}$ is the quantum yield of fluorescence, $\Omega$ is the solid angle of isotropic fluorescence registration.

On the base of the analysis of literature data [6-15], we selected components with the maximum values of fluorescent characteristics. The selected fluorophores and their characteristics are presented in Table 1 . The parameters were obtained from experimental studies of healthy human skin.

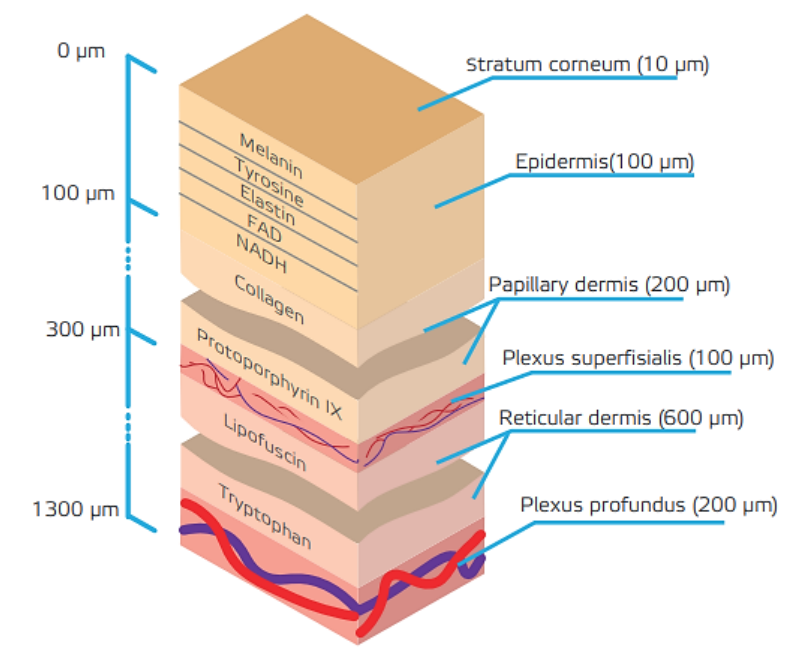

Fig. 1 Fluorophores localization in the six-layer skin model. 
The localization of presented fluorophores in the skin model layers was chosen on the basis of papers review [6-15] is shown in Fig. 1. It is important to note that in all spectral ranges, AF modeling was carried out with fixed values of fluorophore concentrations and their location in the human skin model.

Figs. 2 and 3 show the absorption and emission spectra of selected fluorophores. As can be seen from Fig. 2, the absorption spectra can be divided into three ranges. This is due to the fact that the simulation of fluorescence in different spectral ranges makes it possible to carry out a comprehensive analysis of the skin component composition.

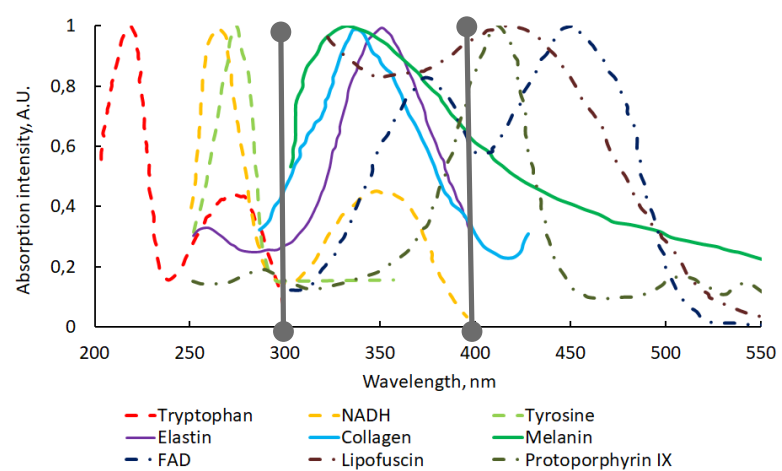

Fig. 2 Absorption spectra of model fluorophores.

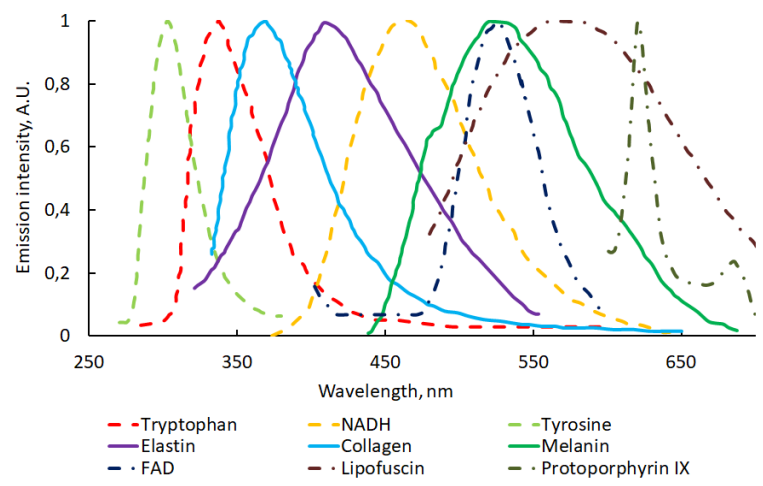

Fig. 3 Emission spectra of model fluorophores.

\section{Results and discussion}

\subsection{Skin model AF excited in the middle UV spectral range $(270-300 \mathrm{~nm})$}

The main objective of this work was fluorescence simulation at different wavelengths with appropriate fluorophores. At the first stage, fluorescence was simulated at excitation wavelengths in the middle UV spectral range $(270-300 \mathrm{~nm})$. The skin model in this range contained five fluorophores that were excited in the UV range: tyrosine, tryptophan, elastin, NADH, Protoporphyrin IX. The results of skin AF simulating excited by different wavelengths from the mid-UV range are presented in Fig. 4.

The maximum intensity of the AF is observed at a wavelength of $340 \mathrm{~nm}$. This is due to the greatest contribution of tryptophan to the fluorescence spectrum, since at the wavelength of $340 \mathrm{~nm}$ there is a fluorescence peak of this component. Although tyrosine at an excitation wavelength of $280 \mathrm{~nm}$ has a stronger absorption than tryptophan, but the indicators of the molar extinction coefficient and the molar concentration are significantly lower than that of tryptophan. As the excitation wavelength increases, the absolute values of AF response declines. This is due to the weakening of the emission signals of the fluorophores, which is confirmed by the emission spectra shown in Fig. 3. The fluorescence intensity decreases with increasing excitation wavelength, without changing the shape or shifting the position of the maximum. A decrease of fluorescence upon excitation with radiation at different wavelengths, controlled by the maximum of fluorescent signal was shown in the study by Brancaleon et al. [16]. Brancaleon et al. obtained similar to our studies results by skin areas irradiating with emitting wavelengths of: $275 \mathrm{~nm}, 285 \mathrm{~nm}, 295 \mathrm{~nm}, 305 \mathrm{~nm}$, and $315 \mathrm{~nm}$.

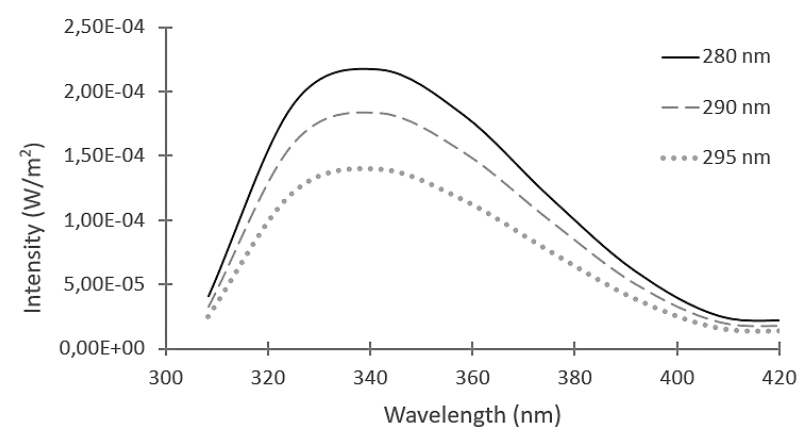

Fig. 4 Model AF spectra of the skin excited by different wavelengths from the mid-UV range.

The efficiency of the model was tested by comparing the simulation results with the experimental data provided by Brancaleon et al. [16]. An excitation wavelength of $295 \mathrm{~nm}$ was chosen. For comparison, the simulation and experimental fluorescence spectra were normalized to a maximum value of intensity (Fig. 5). This approach was also used to test models in other ranges.

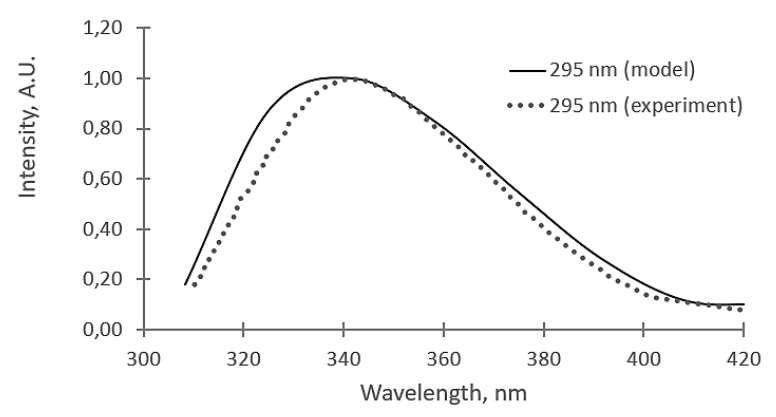

Fig. 5 The simulated and experimental spectra of healthy human skin excited by radiation at wavelength of $295 \mathrm{~nm}$.

Comparing the experimental and modeling spectra reveals small discrepancies with $12 \%$ maximum differences. As can be seen from Fig. 5, the 
experimental spectrum is shifted to the long-wavelength region. This may be due to the fact that not all amino acids were used in the simulation, but only tyrosine and tryptophan. With a larger number of components of this group, the spectrum should be shifted to the longwavelength region by the emission contribution of other fluorophores [17].

\subsection{Skin model AF excited in the near UV spectral range $(330-360 \mathrm{~nm})$}

At the second stage, modeling was performed at excitation wavelengths in the near UV range (330$360 \mathrm{~nm})$. The skin model contained six fluorophores: melanin, elastin, FAD, NADH, collagen, lipofuscin. The skin model fluorescence spectra excited by different wavelengths $(337 \mathrm{~nm}, 350 \mathrm{~nm}, 360 \mathrm{~nm})$ are presented in Fig. 6.

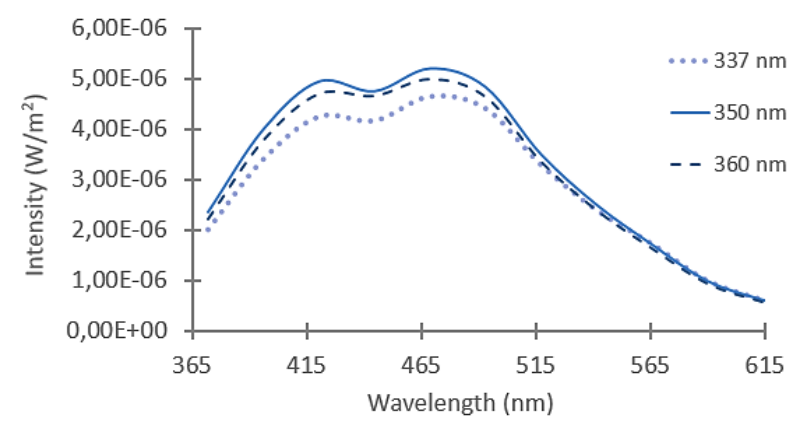

Fig. 6 Model AF spectra of the skin excited by different wavelengths from the near UV range.

The main contribution to the fluorescence spectrum is associated with elastin and FAD. The maximum AF intensity is observed at wavelengths of $415 \mathrm{~nm}$ and $490 \mathrm{~nm}$, which correspond to the emission peaks of elastin and FAD, respectively. The emission peak at wavelength of $415 \mathrm{~nm}$ is explained by the high value of the elastin absorption coefficient at the excitation wavelength, as well as the high values of the quantum yield and molar extinction coefficient compared to other fluorophores. Melanin also contributes to $\mathrm{AF}$ at an emission wavelength of $515 \mathrm{~nm}$, but optical characteristics such as quantum yield and molar extinction coefficient of FAD are several orders of magnitude greater than optical characteristics of melanin.

The model spectrum was compared with experimental results obtained by by Borisova et al. [18]. In Fig. 7 we see the coincidence of the main maxima positions and their ratios. An estimate of the difference between the modeled spectrum and the experimental spectrum was also calculated. The difference is $43 \%$. The peak attributable to elastin in the experimental spectrum is located in long wavelength region. This is due to the fact that the simulation did not take into account tryptophan, which could also lead to changes in the AF spectrum. The $450-550 \mathrm{~nm}$ range of the simulated spectrum is more stretched compared to the experimental one due to the small number of points taken in the simulation and the components variability.

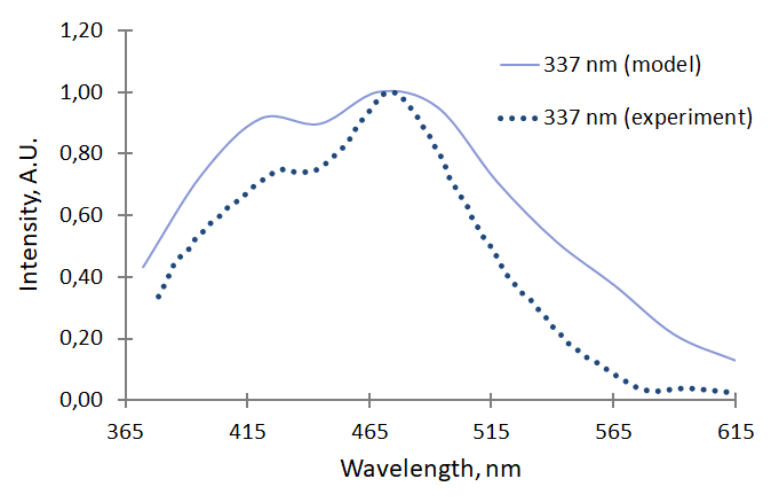

Fig. 7 The simulated and experimental spectra of healthy human skin excited by radiation at wavelength of $337 \mathrm{~nm}$.

\subsection{Skin model AF excited in the visible spectral range $(400-450 \mathrm{~nm})$}

At the last stage, the fluorescence was simulated in the visible range $(380-780 \mathrm{~nm})$ with excitation by radiation with wavelengths of $400-450 \mathrm{~nm}$ (Fig. 8). The skin model contained four fluorophores: melanin, FAD, PP IX, lipofuscin.

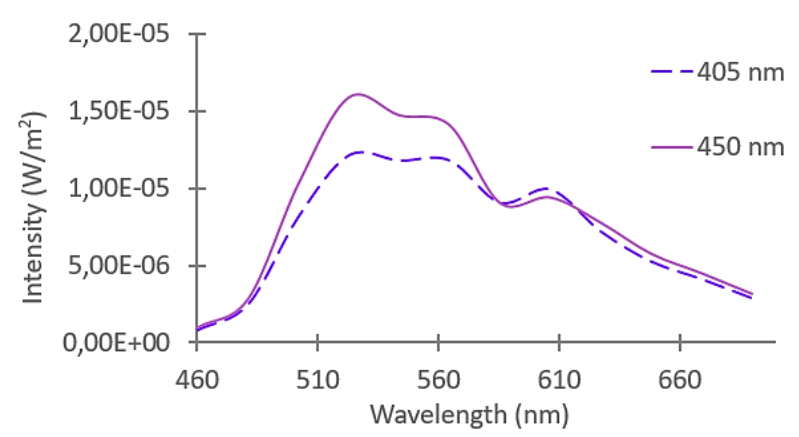

Fig. 8 Model AF spectra of the skin excited by different wavelengths from the visible range.

Peaks of fluorescence are observed at wavelengths of $520 \mathrm{~nm}, 570 \mathrm{~nm}$ and $620 \mathrm{~nm}$. At the peak of $520 \mathrm{~nm}$, FAD makes the largest contribution to $\mathrm{AF}$, since at a given excitation wavelength, this fluorophore has a maximum emission intensity. The peak of the AF spectra at $620 \mathrm{~nm}$ is explained by the largest contribution of lipofuscin at an excitation wavelength of $450 \mathrm{~nm}$, since this component has a higher absorption coefficient than FAD. This fact is confirmed by the graph, where there is a change in the ratios of the fluorescence peaks when excited by radiation of $405 \mathrm{~nm}$ and $450 \mathrm{~nm}$.

A comparison was also made of the model AF spectrum with the experimental data by Borisova et al. upon excitation of skin with $405 \mathrm{~nm}$ radiation [18]. Fig. 9 indicates coincidence of the peaks of the modeled and experimental AF spectra, and the difference in 
fluorescence intensity is no more than $12 \%$. In this case, the differences in the spectra may be due to the variability of the concentrations of fluorophores and the thicknesses of the skin layers and in different locations.

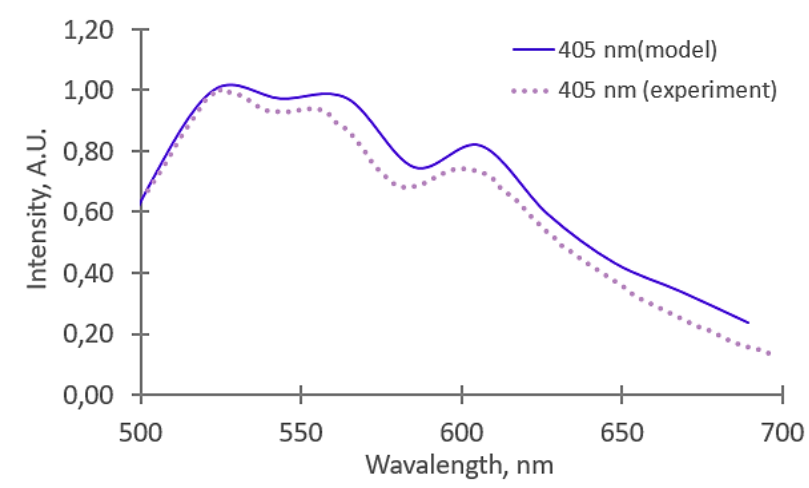

Fig. 9 The simulated and experimental spectra of healthy human skin excited by radiation at wavelength of $405 \mathrm{~nm}$.

\section{Conclusion}

Monte Carlo simulation was used for modelling of human skin AF spectra. Fluorescence was excited by different wavelengths, a change was observed in the absolute values of the fluorescence intensity (near UV range), as well as the shape of the fluorescence spectra (visible range), which coincides with the experimental data of other authors.

The model fluorescence spectrum excited by radiation in the mid-UV range has minimal differences from the experimental spectrum. It was estimated that this difference is $12 \%$. The AF spectrum when excited in the near UV range, obtained by simulation has a difference of $43 \%$. In the visible range, the difference between the spectra is $12 \%$. The reason for the appearance of differences could be the fact that the simulation of fluorescence in different spectral ranges was carried out with fixed values of the concentrations of fluorophores present in the human skin model and their location. The thickness of human skin and the localization of fluorophores can also vary greatly for different samples of skin and various parts of the body. If necessary, the model can be modified for each specific case and the error of the discrepancy with the experimental data can be reduced to a few percent.

As a result of comparing the AF spectra of healthy human skin in three spectral ranges, obtained by simulation and experiment, it can be concluded that the model is correct and that it is possible to use the proposed model for further research and development. The developed skin model can be used in the analysis (interpretation) of AF experimental data and for the devices design for AF measuring (simulation of various excitation and registration optical schemes).

\section{Disclosures}

The author declares that there is no conflict of interests in this paper.

\section{Acknowledgments}

This research was supported by the Ministry of Education and Science of the Russian Federation.

\section{References}

1. A. C. Croce, G. Bottiroli, "Autofluorescence spectroscopy and imaging: A tool for biomedical research and diagnosis," European Journal of Histochemistry 58(4), (2014).

2. V. V. Dremin, A. V. Dunaev, "How the melanin concentration in the skin affects the fluorescence-spectroscopy signal formation," Journal of Optical Technology 83(1), 43-48 (2016).

3. E. G. Borisova, L. P. Angelova, and E. P. Pavlova, "Endogenous and Exogenous Fluorescence Skin Cancer Diagnostics for Clinical Applications," IEEE Journal of Selected Topics in Quantum Electronics 20(2), 211-222 (2014).

4. P. Meredith, J. Riesz, "Radiative Relaxation Quantum Yields for Synthetic Eumelanin," Photochemistry and Photobiology 79(2), 211-216 (2004).

5. V. V. Tuchin, Tissue Optics, Light Scattering Methods and Instruments for Medical Diagnostics, Third Edition, SPIE, Bellingham (2007). ISBN: 9781628415162.

6. M. A. Ansari, E. Mohajerani, "Mechanisms of Laser-Tissue Interaction: I. Optical Properties of Tissue," Journal of Lasers in Medical Sciences 2(3), 119-125 (2011).

7. G. D. Fasman, Handbook of Biochemistry and Molecular Biology, Third Edition, Ohio CRC Press, Cleveland (1976). ISBN 9780367260903.

8. R. F. Chen, "Measurements of absolute values in biochemical fluorescence spectroscopy," Journal of Research of the National Bureau of Standards Section A: Physics and Chemistry 76A(6), 593-606 (1972).

9. I. Meglinski, Biophotonics for Medical Applications, Elsevier, Amsterdam (2014). ISBN: 9780857096623.

10. Collagen alpha-1(I) chain, Protein A005366, Signaling Gateway (date of access: 29.04.2018).

11. Elastin, Protein A002955, Signaling Gateway (date of access: 29.04.2018). 
12. J. Tombran-Tink, C. J. Barnstable, Retinal Degenerations: Biology, Diagnostics, and Therapeutics, Humana Press, Totowa, NJ (2007). ISBN 9781597451864.

13. J. R. Sparrow, Y. Wu, C. Y. Kim, and J. Zhou, "Phospholipid meets all-trans-retinal: the making of RPE bisretinoids," Journal of Lipid Research 51(2), 247-261 (2009).

14. M. Gouterman, G.-E. Khalil, "Porphyrin free base phosphorescence," Journal of Molecular Spectroscopy 53(1), 88100 (1974).

15. K. S. Brandenburg, K. J. Rodriguez, J. F. McAnulty, C. J. Murphy, N. L. Abbott, M. J. Schurr, and C. J. Czuprynski, "Tryptophan inhibits biofilm formation by Pseudomonas aeruginosa," Antimicrobial Agents and Chemotherapy 57(4), 1921-1925 (2013).

16. L. Brancaleon, G. Lin, and N. Kollias, "The In Vivo Fluorescence of Tryptophan Moieties in Human Skin Increases with UV Exposure and is a Marker for Epidermal Proliferation," Journal of Investigative Dermatology 113(6), 977-982 (1999).

17. D. M. Jameson, “A Fluorescent Lifetime: Reminiscing About Gregorio Weber," Chap in Perspectives on Fluorescence, 1-16 (2016).

18. E. Borisova, P. Pavlova, E. Pavlova, P. Troyanova, and L. Avramov, "Optical Biopsy of Human Skin - A Tool for Cutaneous Tumours' Diagnosis," International Journal Bioautomation 16(1), 53-72 (2012). 